\title{
Evaluation of Red Cell Distribution Width and Neutrophil Lymphocyte Ratio as Prognostic Factors in Hypertensive Disease of Pregnancy
}

\section{Mahmoud Sayed ahmed Saad ${ }^{1, *}$ M.Sc.,Emad Abdel-Rahman El-Temamy ${ }^{2}$ MD.,} Ahmed Taha Abdel-Fattah ${ }^{2} \mathrm{MD}$. ,Wael Refaat Hablas ${ }^{3} \mathrm{MD}$.

\author{
*Corresponding Author: \\ Saad, MahmoudSayed \\ dr.mahmoud.saad.1983@gmail.com
}

Received for publication April16, 2020; Accepted May 14, 2020; Published onlineMay 15, 2020.

Copyright 2020 The Authors published by Al-Azhar University, Faculty of Medicine, Cairo, Egypt. All rights reserved. This an openaccess article distributed under the legal terms, where it is permissible to download and share the work provided it is properly cited. The work cannot be changed in anyway or used commercially.

doi:10.21608/aimj.2020.23936.1143

${ }^{1}$ Obstetrics and

GynecologyDepartment, Al-Amreya General Hospital, Alexandria.

${ }^{2}$ Obstetrics and

GynecologyDepartment, Faculty of Medicine, Al-Azhar University Cairo, Egypt.

${ }^{3}$ Clinical pathology Department, Faculty of Medicine, Al-Azhar University Cairo, Egypt.

Disclosure: The authors have no
financial interest to declare in
relation to the content of this
article. The Article Processing
Charge was paid for by the
authors.
$\begin{aligned} & \text { Authorship: All authors have a } \\ & \text { substantial contribution to the article. }\end{aligned}$

\begin{abstract}
Background: Preeclampsia (PET) is characterized by hypertension and proteinuria starting from 20 weeks of gestational age or more with previous normal blood pressure. There are wide varieties of theories causing preeclampsia. The most unbreakable theory is the change of immunological system leading to increased inflammation response.

Aim of the work:To compare red cell distribution width(RDW) and neutrophil lymphocyte ratio(NLR)in normal pregnant women and in women with pregnancy hypertensive disease (PHD) to evaluate whether these parameters have a prognostic significance in diagnosing and determining the severity of PHD.

Patients and methods: A case-control retrospective study was carried out on a total of 536 pregnant women who have met the inclusion criteria with 300 normotensive women (55.97\%) as control and 236 women with PHD (44.03\%) as cases which subdivided into 3 subgroups: de-novo gestational hypertension(G.HTN) $(\mathrm{n}=100)$, mild preeclampsia(PET)(n=50),severe PET and/or eclampsia(n=86).

Results: The mean RDW-coefficient of variation (CV) in the control group was13.83 \pm 0.87 , where in PHD group was15.91 \pm 1.44 with a pvalue of $<0.001$. Also, in the subgroup analysis, there was a difference in mean RDW-CV between women with G.HTN (15.47 \pm 1.21$)$, mild PET $(15.63 \pm 1.18)$ and severe PET $(16.47 \pm 1.63)$ with a p-value of $<0.001$. By analyzing cut-off value $14.8 \%$, RDW-CV had a sensitivity $78.07 \%$ and specificity $88.67 \%$ as a marker for PHD. Also, the mean NLR in the control group was $3.92 \pm 1.83$ where in PHD group was $4.09 \pm 189$ with a p-value of 0.025 . By analyzing cut-off value 5.05, NLR had a sensitivity $33.33 \%$ and specificity $84.67 \%$ as a marker for PHD. Accidentally, it was found that platelet distribution width (PDW) has a considerable significance with a mean of $13.98 \pm 2.72$ in the normotensive group versus $14.82 \pm 2.52$ in the PHD group with a p-value of $<0.001$. Also, in the subgroup analysis, there were significant differences between women with G.HTN (14.04 \pm 2.58$)$, mild PET $(14.92 \pm 1.73)$ and severe PET (15.6 \pm 2.61 ) with a p-value of $<0.001$.

Conclusion: RDW, NLR and PDW can be used as simple, applied, noninvasive and inexpensive parameter for prediction and prognosis of PHD.
\end{abstract}

Keywords: NLR; PDW; PHD; RDW.

\section{INTRODUCTION}

Hypertensive disorders of pregnancy (HDP), including preeclampsia (PET), complicate up to $10 \%$ of pregnancies worldwide, constituting one of the greatest causes of maternal and perinatal morbidity and mortality worldwide.

In the literature, there have been many theories about pathophysiology of PET. Firstly, defective trophoblast invasion was claimed to cause preeclampsia. Another theory was changes in immune system of pregnant women causing increased inflammatory response leading to wrong placentation, increased capillary permeability, microvascular thrombosis, and increased vascular tone. ${ }^{1}$

To date, there have been several studies about predictive markers and prognostic factors of preeclampsia, but unfortunately, only a few have been found to be significant.

The red blood cell distribution width (RDW) is a simple and inexpensive parameter which reflects the degree of heterogeneity of erythrocyte volume (conventionally known as anisocytosis), and is 
traditionally used in laboratory hematology for differential diagnosis of anemias.

Recently,anisocytosis has found to be a commonplace in human disorders such as cardiovascular disease, venous thromboembolism, cancer, diabetes, community-acquired pneumonia, chronic obstructive pulmonary disease, liver and kidney failure, as well as in other acute or chronic conditions. So,RDW has been established its place as a chronic inflammatory marker. ${ }^{2}$

Although the relationship between RDW and hypertension (HTN) has been well documented, there is a lack of data on the association between RDW and preeclampsia. In the present study, we have aimed to investigate the correlation of RDW with preeclampsia and its severity.

Neutrophil is presented as the first line of defense and initiate the non-specific inflammatory mediators, whereas lymphocytes is presented as regulation or protection components of inflammation. Increasing neutrophils are associated with increasing inflammation and on the other hand low levels of lymphocytes are associated with poor general health, increasing cortisol levels, and increasing level of apoptosis due to stressor. So, neutrophil lymphocye ratio (NLR) in complete blood count (CBC) is a nonspecific marker of systemic inflammatory response. ${ }^{3}$

\section{PATIENTS AND METHODS}

This study is a retrospective study where data were collectedfrom the files of the patients in the obstetrics and gynecology department. The data was completed from the data sheet that prepared for every female whoattended AlHusseinUniversityHospital in Cairo during the time period between January 2017 and December 2018.

The data sheets were checked first for the exclusion criteria that included patients with chronic hypertension, either identified before gestation or identified early in pregnancy, patients with hematological conditions associated with increased RDW as iron deficiency anemia, Folate or vitamin B12 deficiency or immune hemolytic anemia, patients with other diseases associated with increased RDW as congestive heart failure and acute myocardial infarction, patients who had received blood transfusion in the last three months before evaluation, patients with severe sepsis or even evidence of any infection as urinary tract infections(UTI)or chorioamnionitis because infection is often associated with leucocytosis which will affect NLR. At last; pregnant women before 20 wks gestation were also excluded.

The total number of the studied group was 536 pregnant women who have been admitted or attended the antenatal clinics. The selected women have been divided into 300 normotensive pregnant women free of any medical disorders as control and patients with pregnancy hypertension disease as cases which were categorized into 3 subgroups: 100 women with de-novo G.HTN, 50 patients with mild PET and 86 patients with severe PET and/or eclampsia.

Clinical definition of severe PET was as follows: a blood pressure (BP) $\geq 160 / 110 \mathrm{mmHg}$ (measured 2 times at least 6 hours apart and less than one week),with either a proteinuria greater than $5 g$ over 24 hours or a urine dipstick showing 3 or 4 in a random urine analysis. Other criteria that may be present: Serum creatinine $>1.2 \mathrm{mg} / \mathrm{dL}$, platelets count $<100.000 / \mathrm{mm}^{3}$, elevated liver enzymes, persistent epigastric pain, persistent headache or other cerebral or visual disturbances, oliguria (urine output $<400 \mathrm{~mL} / 24$ hours), intrauterine growth restriction (IUGR),convulsions (eclamptic fits).

Regarding cases with mild PET, the following criteria are used: $\mathrm{BP} \geq 14090 \mathrm{mmHg}$ and $<160 / 110$ $\mathrm{mmHg}$ and proteinuria $\geq 300 \mathrm{mg}$ and $<5.0 \mathrm{~g} 24$ hours or 1 +dipstick, while non proteinuric G.HTN is diagnosed with $\mathrm{BP} \geq 140 / 90 \mathrm{mmHg}$ with no proteinuria.

In general, the files of pregnant women who had met the inclusion criteria have been checked for the women's age, parity, gestational age, as well as, personal, family, obstetric, medical and surgical history. In addition, vital signs, general, obstetric and local examinations were checked for. Finally, ultrasound examinations and laboratory investigations including:CBC, coagulation profile, complete urine analysis, serum albumin, serum Alanine Aminotransferase (ALT) \& Aspartate Aminotransferase (AST), serum urea, creatinine and serum uric acid(SUA).

\section{Statistical methodology:}

The collected data was organized, tabulated and statistically analyzed using Statistical Package for Social Science (SPSS) version 16 (SPSS Inc. Illinois, Chicago, USA), running on IBM compatible computer with the help of Microsoft Office Excel 2007.

RDW and NLR were measured using SYSMEX and SIEMENS ADVIA 120 Hematology system.

For quantitative data: mean, median, standard deviation (SD) and range (minimum-maximum) were calculated and for comparison between groups the following tests were used: ANOVA test, Post Hoc Test, Student's t-test, Kruskal Wallis test and Mann Whitney test.

For qualitative data: frequency and percent distribution were calculated and for comparison between groups, chi square test was calculated.

For interpretation of results, $\mathrm{p}$ value was evaluated as follows: P-value $<0.05$ was considered significant, $\mathrm{P}$-value $<0.001$ was considered as highly significant while P-value $>0.05$ was considered insignificant. 
The prognostic value of RDW and NLR were evaluated in combination with clinical, radiological and laboratory assessment of the cases to help in development of predictive and diagnostic assay of PHD.

RDW-CV in human RBC is $11.5-14.5 \%$ where it is mathematically calculated with the following formula: RDW-CV=(Standard deviation of Mean Corpuscular Volume(MCV) $\div$ mean MCV) $\times 100$, while normal reference range of NLR is 1.2-4.4.

\section{RESULTS}

In the present study, a total of 536 pregnant women who have met the inclusion criteria were included with 300 normotensive pregnancies (55.97\%) and 236 patients with PHD (44.03\%).The mean maternal age of the normotensive group was $27.75 \pm 5.69$ years while the mean maternal age of the hypertensive group was $30.70 \pm 6.47$ years. The baseline characteristics of normotensive pregnant women and patients with PHD are summarized in (Table1).

RDW CV, RDWSD, NLR, platelet distriution width (PDW), systolic BP, diastolic BP, SUA were found to be possible predictors of PET occurrence. All these parameters were found to be higher with increased severity of the disease except NLR which was associated only with occurrence but not with the severity of the condition.

In our study, receiver operating characteristic (ROC) curve analysis showed reliable diagnostic significance of RDW-CV with area under the curve (AUC) of 0.898 (95\%Confidence Interval (CI) 0.8690.927) as shown on (Figure 1).By analyzing cut-off value 14.8 , RDW-CV had a sensitivity $78.07 \%$ and specificity $88.67 \%$ as a marker for PHD.

Similarly, RDW-SD showed reliable diagnostic significance with AUC 0.829 (95\% CI 0.791-0.866) as shown on (Figure 2). By analyzing cut-off value 46.3, RDW-SD had a sensitivity $68.42 \%$ and sensitivity $86 \%$ as a marker for HDP.

\begin{tabular}{|c|c|c|c|c|c|}
\hline Variables & \begin{tabular}{|l|} 
Normotensive \\
$(\mathrm{n}=300)($ Mean \\
$\pm \mathrm{SD})$
\end{tabular} & \begin{tabular}{|l|} 
G. HTN \\
$(n=100)($ Mean \pm \\
SD)
\end{tabular} & \begin{tabular}{|l|} 
Mild PET \\
$(n=50)($ Mean \pm \\
SD)
\end{tabular} & \begin{tabular}{|l|} 
Severe PET \\
$(\mathrm{n}=86)($ Mean \pm \\
$\mathrm{SD})$
\end{tabular} & Pvalue \\
\hline Age (years) & $27.75 \pm 5.69$ & $33.11 \pm 6.18$ & $27.78 \pm 6$ & $29.81 \pm 6.17$ & 0.230 \\
\hline $\begin{array}{l}\text { Gestational } \\
\text { age } \\
\text { (weeks) }\end{array}$ & $33.34 \pm 4.26$ & $35.21 \pm 3.11$ & $35.02 \pm 3.79$ & $33.14 \pm 4.66$ & 0.340 \\
\hline $\begin{array}{l}\text { Parity } \\
P G \\
M G\end{array}$ & & \begin{tabular}{|l}
11 \\
89
\end{tabular} & $\begin{array}{l}15 \\
35\end{array}$ & $\begin{array}{l}23 \\
63\end{array}$ & 0.140 \\
\hline $\begin{array}{l}\mathrm{SBP} \\
(m m \mathrm{Hg})\end{array}$ & $105.14 \pm 13$ & $151.83 \pm 10.5$ & $146.6 \pm 6.6 .8$ & $164.2 \pm 15.1$ & $<0.001^{\circ}$ \\
\hline $\begin{array}{l}\text { DBP } \\
(m m ~ H g)\end{array}$ & $74.3 \pm 7$ & $96.34 \pm 5.26$ & $93.7 \pm 7.7$ & $100.83 \pm 8.15$ & $20.001^{\prime}$ \\
\hline $\begin{array}{l}\text { Fetal } \\
\text { gender } \\
\text { Male ratio } \\
\text { Female ratio }\end{array}$ & $\begin{array}{l}52 \%(n=156) \\
48 \%(n=144)\end{array}$ & $\begin{array}{l}52 \%(n=52) \\
48 \%(n=48)\end{array}$ & $\begin{array}{l}58 \%(n=29) \\
42 \%(n=21)\end{array}$ & $\begin{array}{l}60.6 \%(n=52) \\
39.53 \%(n=34)\end{array}$ & 0.522 \\
\hline $\begin{array}{l}\text { S. Albumin } \\
\text { ( }(\mathrm{g} / \mathrm{dL})\end{array}$ & $3.37 \pm 0.48$ & $3.35 \pm 0.49$ & $3.77 \pm 0.84$ & $3.22 \pm 0.47$ & 0.130 \\
\hline $\begin{array}{l}\text { SUA } \\
\text { (mg/dL) }\end{array}$ & $4.47 \pm 1.77$ & $4.84 \pm 1.25$ & $5.10 \pm 0.84$ & $5.5 \pm 1.92$ & $80.001^{\circ}$ \\
\hline $\begin{array}{l}\text { Platelets } \\
\times 1000\end{array}$ & $223.75 \pm 63.82$ & $209.56 \pm 69.15$ & $229.65 \pm 66.65$ & $230.57 \pm 68.59$ & 0.150 \\
\hline PDW & $13.98 \pm 2.72$ & $14.04 \pm 2.58$ & $14.92 \pm 1.73$ & $15.60 \pm 2.61$ & $<0.001^{\prime}$ \\
\hline $\begin{array}{l}\text { Birth } \\
\text { weight }(k g)\end{array}$ & $2.92 \pm 64$ & $2.86 \pm 0.67$ & $2.57 \pm 0.78$ & $2.23 \pm 0.7$ & $<0.001$ \\
\hline NLR & $3.92 \pm 1.83$ & $3.92 \pm 1.79$ & $4.03 \pm 1.36$ & $4.31 \pm 2.22$ & 0.097 \\
\hline
\end{tabular}

Normal reference range of RDW-standard deviation (SD) in human red blood corpuscles (RBC) is $39-46 \mathrm{fL}$ and normal reference range of

Table 1: Baseline characteristics of normotensive pregnant women and patients with PHD.

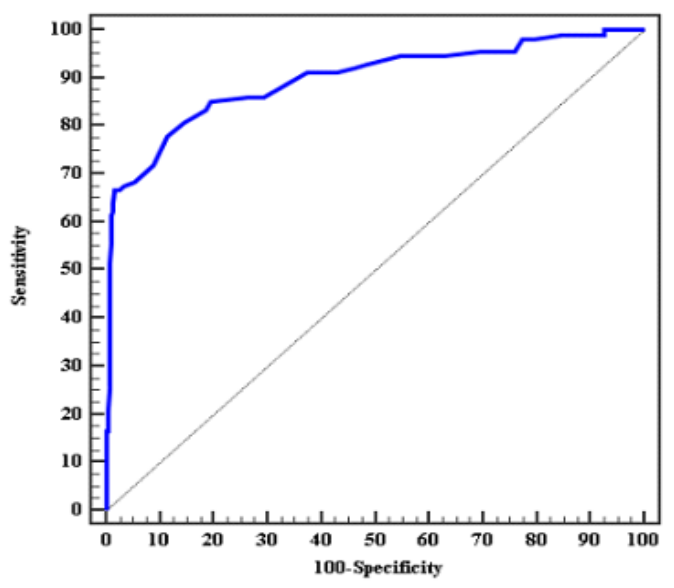

Fig. 1: ROC curve for RDW CV to diagnose women with PHD from normotensive women.

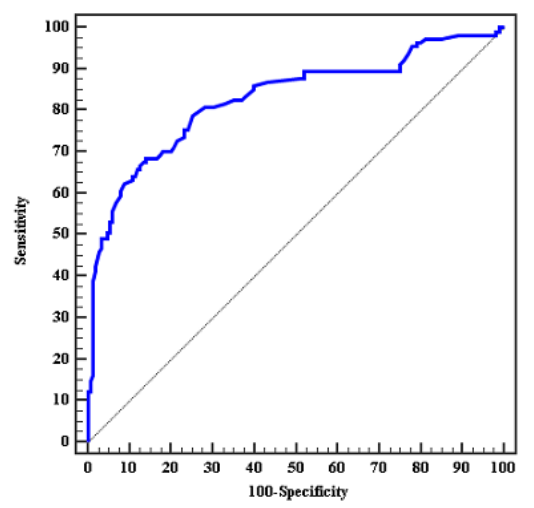

Fig. 2: ROC curve for RDW SD to diagnose PHD from normotensive women.

NLR showed weaker significance in ROC curve analysis with AUC 0.557 (95\% CI 0.506-0.607) as shown on (Figure 3). By analyzing cut-off value 5.05 ,NLR had a sensitivity $33.33 \%$ and specificity $84.67 \%$ as a marker for HDP.

Accidently discovered in our study, ROC curve analysis showed reliable diagnostic significance of PDW with AUC 0.580 (95\% CI 0.532-0.629)as shown on (Figure 4). By analyzing cut off value 12 femtolitre(fL), PDW had a sensitivity of $91.23 \%$ and specificity of $31.67 \%$ as a marker for PHD. 


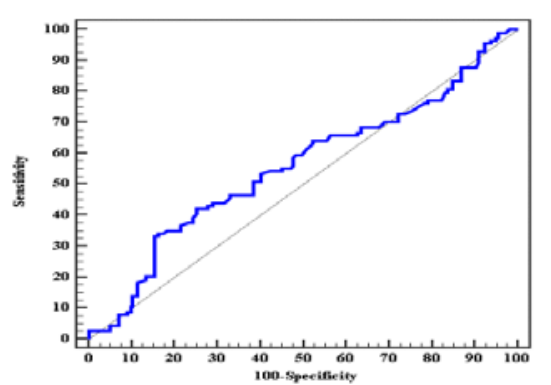

Fig. 3:ROC curve for NLR to diagnose PHD from normotensive women.

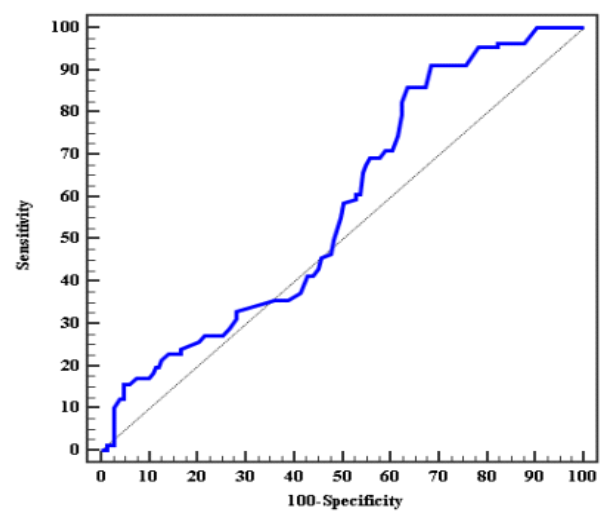

Fig. 4: ROC curve for PDW to diagnose PHD from normotensive women.

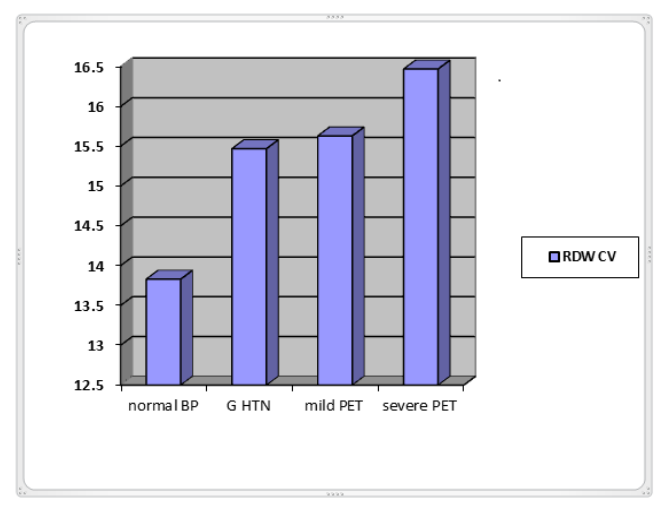

Fig. 5: Comparison between the four studied groups according to RDW CV mean.

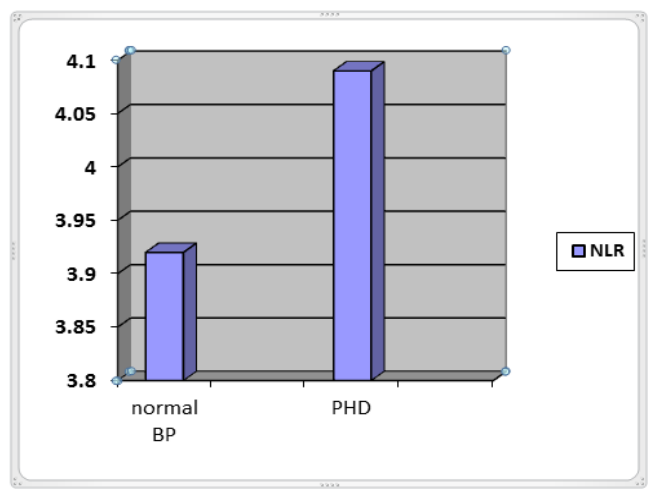

Fig. 6:Comparison between the normotensive and PHD groups according to NLR mean

\section{DISCUSSION}

Several studies have evaluated the role of biochemical markers or a combination of biochemical and biophysical markers in the prediction of preeclampsia. Regardless of the parameters used, screening for preeclampsia in low risk women is associated with very low positive predictive values ranging from $8 \%$ to $33 \%$. So in presence of one or more risk factor, the positive predictive value (PPV) of the used test is increased. ${ }^{4}$

There was a significant difference between the studied groups regarding arterial blood pressure both systolic and diastolic (P-value $<0.001)$ with a mean SBP and DBP of $154.2 \pm 10.8 \mathrm{mmHg}$ and $97 \pm 7 \mathrm{mmHg}$ respectively in the hypertensive group. On the other hand, mean SBP of $105.14 \pm 13 \mathrm{mmHg}$ and mean DBP of $74.3 \pm 7 \mathrm{mmHg}$ were found in the normal group.

With severe PET, mean systolic blood pressure (SBP) and diastolic blood pressure (DBP) were higher than those of mild PET(164.2 $\pm 15.1 \mathrm{mmHg}$ and $100.83 \pm 8.15 \mathrm{mmHg}$ versus $146.6 \pm 6.8$ $\mathrm{mmHg}$ and $93.7 \pm 7.7 \mathrm{mmHg}$ respectively).

Of interest, mean SBP and DBP in G.HTN group $(151.83 \mathrm{mmHg} \pm 10.5 \& \quad 96.34 \pm 5.26 \quad \mathrm{mmHg}$ respectively) was higher than those of mild PET, but still lower than severe PET group. Buchbinder A et al. recommended that patients with severe gestational HTN require management similar to those with severe PET because they have worse perinatal outcomes than women with mild preeclampsia. ${ }^{5}$

The main concern in the study was to determine a simple, applied, non-invasive and inexpensive measure to predict the occurrence and severity of PHD.

Elevated RDW level has been used as a marker for increased systemic inflammation. Recent studies have reported that high RDW level is also associated with poor prognosis in various clinical circumstances such as heart failure and coronary artery diseases. The possible explanation of this correlation was made by accusing chronic inflammation and increased inflammatory activity in these conditions. ${ }^{6}$

In our study, there was a difference in mean RDW between women with G.HTN, mild PET and severe PET(p-value $<0.001$ ) which reflect that RDW was associated not only with the presence of PET, but also with its severity.

These results are consistent with a study which had been performed by Y1lmaz ZV and colleagues on 118 pregnant women with preeclampsia and 120 uncomplicated pregnant women which were included in the study. The RDW values were significantly higher in PET group compared with the control group $(15.23 \pm 1.96$ vs.14.48 \pm 1.70 , $\mathrm{p}<0.05)$. The study also confirmed that RDW levels were significantly higher in severe PET group $(15.92 \pm 1.99)$ than mild PET group $(15.08 \pm 2.07)$ in subgroup analysis $(\mathrm{p}<0.05)$. These results are similar 
to our study where the average RDW-CV in mild and severe PET were $15.63 \pm 1.18$ and $16.47 \pm 1.63$ respectively. $^{7}$

Gezer et al. concluded that RDW is associated with the severity of hypertension. RDW predicts a poor prognosis of hypertension leading to heart failure and coronary heart disease. This study revealed that RDW was increased in chronic inflammation situation. Reddy et al. were also reported that RDW is useful to differentiate between severe preeclampsia and mild preeclampsia. However we found that away from their study, RDW is more specific as a marker for preeclampsia. ${ }^{2}$

All anemic cases were excluded from our study since decreased $\mathrm{Hb} \%$ is known to elevate RDW. This is opposite to the study done by Hkima F in a case-control study on Syrian pregnant women. The results of this study showed that mean RDW value in pregnant women with preeclampsia (when anemic and non-anemic patients were merged together) was higher than those of controls(pvalue $=0.0001$ )as well as in preeclampsia patients who were not anemic $(\mathrm{Hb} \geq 11 \mathrm{~g} / \mathrm{dl}) \quad(\mathrm{p}$ value $=0.0001$ ). This suggests that high RDW in preeclampsia patients may be not associated with anemia. This finding may be explained by an effect modification phenomenon between anemia and preeclampsia on RDW values. $^{8}$

NLR in CBC panels is a nonspecific marker of systemic inflammatory response. In our study, significant differences in NLR were found between PHD and normal pregnancy groups. This is in accordance with several previous studies which concluded that NLR has a prognostic value to predict diseases such as malignancy, coronary heart disease and PET.

Oylumlu et al. have reported higher neutrophil counts and NLR in preeclamptic patients, implying that there is an increased inflammatory state. ${ }^{9}$ However, contrary to our findings, Myatt et al. have reported no significant difference in NLR in patients with PET. ${ }^{10}$

Although NLR is higher with increased severity of the disease, this increase is not a statistically significant(p-value of 0.097).

In addition to their capacity to release pre-stored inflammatory mediators, platelets can also produce eicosanoids, such as thromboxane A2, prostaglandin F2 alpha(PGF2a), and PGE2,to regulate inflammatory response and can synthesize peptide mediators, such as interleukin(IL)-1ßon stimulation. Changes in PDW have been described in various autoimmune and inflammatory diseases where significant correlation between high PDW and increased disease activity was found in rheumatoid arthritis, ankylosing spondylitis, ulcerative colitis and chronic obstructive pulmonary disease. $^{11}$

In our study, the increase in the PDW was greater in pregnant women with PET than in healthy pregnant women. The platelet activation in PET is related to alteration of the coagulation process between the platelets and endothelial cells. Basic research has demonstrated that plasma thrombopoietin increases in patients with PET as a major marker of platelet activity $^{12}$.

Therefore, as a parameter for platelet activation, PDW is increased in PET more than in normal pregnant women. These results suggest an active turnover of platelet production in the bone marrow that results in continuous platelet consumption in peripheral blood, and is in accordance with results from previous studies such as that did by Yang SW et al, which declared that among the evaluated platelet indices, only the PDW was observed to have a statistically significant correlation with the mean arterial pressure, which is the best-known severity marker of PET. The ROC curve analysis of the platelet indices in the PET group identified the PDW value of $>13.5 \mathrm{fL}$ as the optimal cut-off level for the prediction of severity. This value had an AUC of 0.74 , a sensitivity of $72 \%$,a specificity of $71 \%$, a PPV of $70 \%$, and a negative predictive value of $71 \%$ (Figure7).

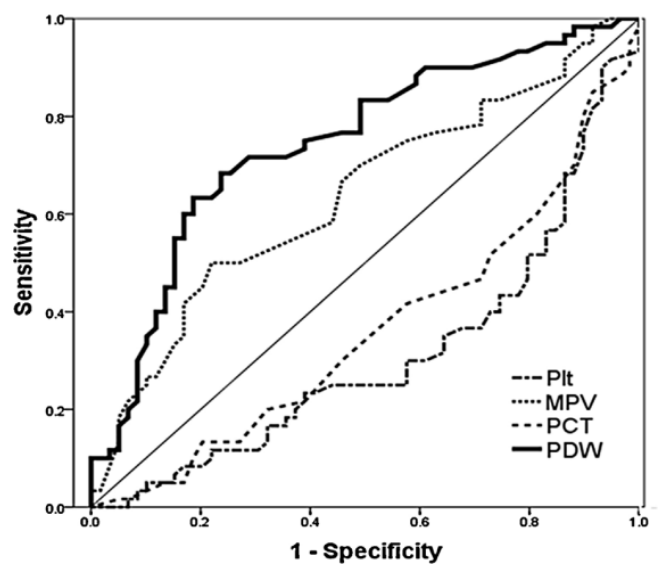

Fig. 7:ROC curve for of platelet indices in the preeclampsia (PET) group to identify the optimal cut-off level for the prediction of the severity of PET (Yang SW et al.,2014).

This result is similar toour study which decided that byanalyzing cut off value $12 \mathrm{fL}$, PDW had a sensitivity of $91.23 \%$ and specificity of $31.67 \%$ as a marker for PHD. ${ }^{13}$

According to Yang SW et al. platelet count is not an absolute indicator for the progression of PET, and the alteration of platelet function and activity should be evaluated. These results are similar to our study where the mean platelet count is nearly equal in the 2 groups $(223.75 \pm 63.82 \times 1000$ in normotensive, $222.42 \pm 68.82 \times 1000$ in PHD) with a p-value of 0.821 which is statistically not significant. ${ }^{13}$

In our study, most of PHD patients were multigravidas 187 (79.2\%) while 49 (20.8\%) were primigravida. Also,the mean age of hypertensive group was $30.70 \pm 6.47$ years while the mean age of normotensive group was $27.75 \pm 5.69$. Although very young primigravida is more susceptible to PET and PET is generally considered to be a disease of women who are pregnant for the first time, recurrence rate in subsequent pregnancy is relatively 
high in addition to that the incidence of PET increases with advanced maternal age so, the prevalence of PET in our study is higher in multigravida. This result is in accordance with a cohort study that was conducted by Mostello D et al. on 6157 women with preeclampsia, in which $14.7 \%$ women with prior preeclampsia developed recurrent preeclampsia and another retrospective study that was conducted by Surapaneni T et al. on 82 women with previous PET, in whom 22 women (about 25\%) had developed recurrent PET. ${ }^{14}$

Uric acid is a marker of oxidative stress, tissue injury and renal dysfunction and it has been used as a marker for severity of preeclampsia. Managing 445 women with severe preeclampsia and eclampsia, Wiltin et al. concluded that a serum uric acid (SUA) concentration increases with an elevation in both SBP\&DBP and a level of $8.1 \mathrm{mg} / \mathrm{dl}$ is associated with increased incidence of eclampsia, in preeclamptic patients.

In our study, marked elevations of SUA occurs with increased severity of the disease with a p-value of $<0.001$. The mean SUA in the normotensive group was $4.47 \pm 1.77 \mathrm{mg} / \mathrm{dl}$ while that of hypertensive group was $5.15 \pm 1.50 \mathrm{mg} / \mathrm{dl}$. Also mean SUA increases with increased severity of PHD $(4.84 \pm 1.25 \mathrm{mg} / \mathrm{dl}$ for G.HTN, $5.10 \pm 0.84 \mathrm{mg} / \mathrm{dl}$ for mild PET,5.5 $\pm 1.92 \mathrm{mg} / \mathrm{dl}$ for severe PET).

Increased SUA in PET is due to many reasons; firstly, as a result of a decrease in SUA clearance that is secondary to fall in glomerular filtration rate(GFR)due to vasoconstrictors such as angiotensin II, nor epinephrine and endothelin. Also, elevated lactic acid levels produced by hypoxic placenta are reported in PET as hyperlactacidemia interferes with uric acid excretion. Furthermore, PET is associated with increased free radical formation and elevated oxidative stress. SUA production is coupled with formation of reactive oxygen species when the Xanthine oxidase/dehydrogenase enzyme is in the oxidase form. ${ }^{15}$

For the past two decades, there have been conflicting reports in the literature regarding usefulness of SUA estimation in preeclamptic women. The present study results may attempt to reappraise its role in evaluating the severity of preeclampsia.

According to serum albumin, there were no statistically significant differences between the studied group $(3.37 \pm 0.48 \mathrm{mg} / \mathrm{dl}$ in normotensive Vs. $3.40 \pm 0.61 \mathrm{mg} / \mathrm{dl}$ in PHD group)

In our study, the average birth weight is lowered with increased severity of the disease $(2.86 \pm 0.67 \mathrm{~kg}$, $2.57 \pm 0.78 \mathrm{~kg}, 2.23 \pm 0.7 \mathrm{~kg}$ ) in G.HTN, mild PET, severe PET groups respectively with stillbirth rate about $61.79 \%$ in women with severe PET(55 out of 89).

According to fetal gender, male to female ratio in the normotensive group was 52\%:48\% while in the PHD group was 56.4\%:43.6\%.The percentage ofmale fetus in PHD group was slightly higher but not statistically significant with a p-value of 0.351 . Kaskolka D et al. have idetified 534 studies yielding data on 3,161,735 women. Most of these studies showed no association between male fetal sex and maternal risk of preeclampsia/eclampsia (RR 1.0;95\% CI 0.79-1.05).However, a subgroup analysis including only studies that evaluated nonasian population demonstrated that male fetal sex was associated with increased maternal risk of preeclampsia/eclampsia (RR 1.05;95\% CI 1.031.06). ${ }^{16}$

\section{CONCLUSION}

Unlike many other inflammatory markers and bioassays, RDW, NLR and PDW are inexpensive and readily available new markers that may be useful for prediction of occurrence of PHD. Nevertheless, only RDW and PDW can be used for assessing the severity of the condition.

\section{REFERENCES}

1. Saito S, Shiozaki A, Nakashima A, et al. The role of the immune system in preeclampsia. Mol Aspects Med 2007; 28: 192-209.

2. Gezer C, Ekin A, Solmaz U, et al. MÖzeren M. The value of red cell distribution width for predicting subsequent preeclampsia. Cukurova Med J. 2016;41:224-8.

3. Mehmet EA, Mehmet ZB andHalit A. Mean platelet volume and neutrophil lymphocyte ratio as new markers of preeclampsia severity. J Koşuyolu Heart. 2015;18: 84-88.

4. ACOG Practice Bulletin No.202. Gestational Hypertension and Preeclampsia. Obstet Gynecol. 2019;133(1):e1-e25.

5. Buchbinder A, Sibai BM, Caritis S, et al. Adverse perinatal outcomes aresignificantly higher in severe gestational hypertension than in mild preeclampsia. Am JObstet Gynecol. 2002;186:66-71.

6. Kurt RK, Aras Z, Silfeler DB, et al. Relationship of red cell distribution width with preeclampsia. pregnancyhypertens. 2016 Oct;6(4):285-287.

7. Yılmaz ZV, Yılmaz E andKüçüközkan T. Red blood cell distribution width: A simple parameter in presence and severity of preeclampsia. ClinApplThrombHemost. 2015;21(2):128-31.

8. Hkima F. Relationship of red cell distribution width with preeclampsia in syrian pregnant women. J Arab B H Specializations. 2017; 18:27-34. 
9. Oylumlu M, Ozler A, Yildiz A, et al. New inflammatory markers in pre-eclampsia: echocardiographic epicardial fat thickness and neutrophil to lymphocyte ratio. ClinExpHypertens. 2014;36(7):503-7.

10. Myatt L, Clifton RG, Roberts JM, et al. Firsttrimester prediction of preeclampsia in nulliparous women at low risk. Obstet Gynecol. 2012;119:1234-42.

11. Freitas LG, Alpoim PN, Komatsuzaki F, et al. Preeclampsia. are platelet count and indices useful for its prognostic. Hematology. 2013;18:360-4.

12. Piazze J, Gioia S, Maranghi L, et al. Mean platelet and red blood cell volume measurements to estimate the severity of hypertension in pregnancy. $J$ Perinat Med. 2006;34:246-7.
13. Yang SW, Cho SH, Kwon HS, et al. Significance of the platelet distribution width as a severity marker for the development of preeclampsia. Eur $J$ ObstetGynecolReprod Biol. 2014; 107-111.

14. Surapaneni T, Patil V, and Praveen C. Risk for Recurrence of Pre-eclampsia in the Subsequent Pregnancy. J ClinDiagn Res. 2013; 7(12): 2889-2891.

15. Nair A andSavitha C. Estimation of Serum Uric Acid as an Indicator of Severity of Preeclampsia and Perinatal Outcome. $J$ ObstetGynecol India. 2017; 67(2):109-118.

16. Kaskolka D, Retnakaran R, Zinman B, et al. Fetal sex and maternal risk of preeclampsia / eclampsia: a systematic review and metaanalysis. BJOG. 2017;124(4):553-560. 